\title{
Taming the zoo of supersymmetric quantum mechanical models
}

\author{
A.V. Smilga ${ }^{1}$ \\ SUBATECH, Université de Nantes, \\ 4 rue Alfred Kastler, BP 20722, Nantes 44307, France \\ E-mail: smilga@subatech.in2p3.fr
}

ABSTRACT: We show that in many cases nontrivial and complicated supersymmetric quantum mechanical (SQM) models can be obtained from the simple model describing free dynamics in flat complex space by two operations: (i) Hamiltonian reduction and (ii) similarity transformation of the complex supercharges. We conjecture that it is true for any SQM model.

KEYwords: Field Theories in Lower Dimensions, Extended Supersymmetry, Differential and Algebraic Geometry

ARXIV EPRINT: 1301.7438

\footnotetext{
${ }^{1}$ On leave of absence from ITEP, Moscow, Russia.
} 


\section{Contents}

1 Introduction $\quad 1$

$2 \mathcal{N}=2$ sigma models $\quad 3$

2.1 Dolbeault complex 3

2.2 De Rham complex 5

$\begin{array}{lll}3 & \text { Extended supersymetries } & \mathbf{7}\end{array}$

3.1 Kähler sigma models $\quad 7$

3.2 Hyper-Kähler sigma models $\quad 9$

3.3 HKT and OKT 9

3.4 Reduced models 11

4 Gauge models $\quad 13$

$\begin{array}{lll}5 & \text { Field theories } & \mathbf{1 5}\end{array}$

$\begin{array}{lll}6 & \text { Discussion and outlook } & 17\end{array}$

A Kähler and hyper-Kähler superalgebras $\quad 18$

\section{Introduction}

It is a common belief now that whatever the Grand Unified Theory is, it is a version of supersymmetric field theory. Incorporating supersymmetry is the only known natural way to resolve the hierarchy problem.

An excellent playground to study supersymmetric dynamics is provided by SQM models. The simplest nontrivial such model introduced in [1] has the supercharges

$$
Q=\psi\left[p+i W^{\prime}(x)\right], \quad \bar{Q}=\bar{\psi}\left[p-i W^{\prime}(x)\right]
$$

and the Hamiltonian

$$
H=\frac{1}{2}\left[p^{2}+\left(W^{\prime}\right)^{2}+W^{\prime \prime}(x)(\bar{\psi} \psi-\psi \bar{\psi})\right] .
$$

In classical theory, $p, x$ are the usual conjugated phase space variables and $\bar{\psi}, \psi$ are canonically conjugated Grassmann variables. In quantum theory (which we will be mainly concerned with), $x$ and $\psi$ are still usual real and complex Grassmann numbers, while $p$ and $\bar{\psi}$ become differential operators, $p=-i \partial / \partial x, \bar{\psi}=\partial / \partial \psi$. 
$W(x)$ is an arbitrary function. The operators (1.1), (1.2) satisfy the simplest supersymmetry algebra,

$$
Q^{2}=\bar{Q}^{2}=0, \quad\{\bar{Q}, Q\}=2 H .
$$

Since [1], a lot of other models have been constructed. Many such models have a rather complicated form. Some of them have extended supersymmetries - several pairs of complex supercharges $Q_{a}, \bar{Q}_{a}$ satisfying the algebra ${ }^{1}$

$$
\left\{Q_{a}, Q_{b}\right\}=0, \quad\left\{Q_{a}, \bar{Q}_{b}\right\}=2 \delta_{a b} H, \quad a, b=1, \ldots, \frac{\mathcal{N}}{2} .
$$

The models involving up to 8 such pairs are known.

One can write the action as an integral over the usual $(t, \theta, \bar{\theta})$ or extended $\left(t, \theta_{j}, \bar{\theta}_{j}\right)$ superspace of a usual or extended real superfield expressed via fundamental superfields. Such action is manifestly invariant under supersymmetry transformations. The problem is, however, that many such superfields and many such invariant actions can be constructed.

In this paper, we suggest an alternative approach. Instead of working in superspace, we restrict ourselves with supercharges and Hamiltonians expressed in components. Then we observe that, in the all studied cases, an SQM system can be obtained from the basic simple system describing the free flat complex dynamics,

$$
Q=\sqrt{2} \psi_{a} \pi_{a}, \quad \bar{Q}=\sqrt{2} \bar{\psi}_{a} \bar{\pi}_{a}, \quad \begin{aligned}
& H=\bar{\pi}_{a} \pi_{a}, \\
& (a=1, \ldots, d),
\end{aligned}
$$

where $d$ is the complex dimension.

It is achieved by a combination of two operations: (i) Hamiltonian reduction and (ii) similarity transformation of supercharges.

As a warm-up, let us obtain in this way the model (1.1), (1.2). We start from the model (1.5) with $d=1$. The complex momentum $\pi$ has the real and imaginary parts, $\pi=\left(p_{x}+i p_{y}\right) / \sqrt{2}$. The wave functions depend on $x, y$ and the Grassmann holomorphic variable $\psi$. At the first step, we impose the constraint $p_{y} \Psi=-i \partial \Psi / \partial y=0$. We are allowed to do it as $p_{y}$ commutes with the Hamiltonian. The reduced Hamiltonian is just $p_{x}^{2} / 2$.

The constraint commutes not only with the Hamiltonian, but also with the supercharges. This implies that the reduced system enjoys the same $\mathcal{N}=2$ supersymmetry as the parent one. The reduced supercharges are

$$
Q^{\text {free }}=p_{x} \psi, \bar{Q}^{\text {free }}=p_{x} \bar{\psi} .
$$

It is just the free $(W=0)$ version of Witten's model (1.1), (1.2).

The potential can be introduced at the second step by a similarity transformation. Indeed, the supercharges (1.1) can be expressed via the free ones as

$$
Q=e^{W} Q^{\text {free }} e^{-W}, \quad \bar{Q}=e^{-W} \bar{Q}^{\text {free }} e^{W} .
$$

\footnotetext{
${ }^{1}$ Following the commonly adopted nowadays convention, $\mathcal{N}$ denotes the total number of real conserved supercharges. For the models with physical supersymmetry of the spectrum that involve (at least) double degeneracy of all excited states, $\mathcal{N}$ is always even.
} 
The transformed supercharges $Q, \bar{Q}$ are nilpotent if $Q^{\text {free }}, \bar{Q}^{\text {free }}$ are nilpotent and they hence satisfy the same supersymmetry algebra. Note that this is a similarity transformation for the supercharges. The operator $e^{W}$ is not unitary such that $Q$ and $\bar{Q}$ are transformed in a different way. As for the Hamiltonian $\{\bar{Q}, Q\}$, it is not related to the free Hamiltonian by any similarity transformation and has a distinct spectrum.

This example is trivial, but we will see in the next two sections that this philosophy works in many other not so trivial cases and a complicated SQM model can be obtained from the free model (1.5) by performing a proper Hamiltonian reduction and a proper similarity transformation.

\section{$2 \mathcal{N}=2$ sigma models}

\subsection{Dolbeault complex}

Let us concentrate on $Q$ and perform the following similarity transformation of the free supercharge in (1.5),

$$
Q=e^{R} Q^{\text {free }} e^{-R}
$$

where $R$ is not just a function of coordinates as in (1.6), but an operator, $R=\omega_{a b} \psi_{a} \bar{\psi}_{b}$. The supercharge $\bar{Q}$ will then be rotated with the operator $e^{-R^{\dagger}}$. (It will be convenient for us later to introduce $\bar{Q}$ rotated with an extra scalar function reflecting the presence of a nontrivial Hilbert space measure in the rotated system — see eq.(2.10) below. But let us keep for a moment $Q$ and $\bar{Q}$ Hermitially conjugate in the naive sense, without taking into account the measure.) When $\omega_{a b}$ is anti-Hermitian, $e^{R}$ is unitary, $Q, \bar{Q}$, and $H$ are rotated by the same operator, and this boils down to a canonical transformation of the phase space variables. On the other hand, when $\omega_{a b}$ is Hermitian, the supercharges $Q$ and $\bar{Q}$ are transformed differently, and their anticommutator is nontrivial.

The calculation can be done using the Hadamard formula,

$$
e^{R} X e^{-R}=X+[R, X]+\frac{1}{2}[R,[R, X]]+\ldots
$$

In our case, this implies

$$
\begin{aligned}
e^{R} \psi_{c} e^{-R} & =\psi_{a}\left(e^{\omega}\right)_{a c} \\
e^{R} \partial_{c} e^{-R} & =\partial_{c}+\left(e^{\omega}\right)_{a e}\left(\partial_{c} e^{-\omega}\right)_{e b} \psi_{a} \bar{\psi}_{b} .
\end{aligned}
$$

We thus derive

$$
Q=\sqrt{2} \psi_{d}\left(e^{\omega}\right)_{d c}\left[\pi_{c}-i\left(e^{\omega}\right)_{a e}\left(\partial_{c} e^{-\omega}\right)_{e b} \psi_{a} \bar{\psi}_{b}\right]
$$

The associated Hamiltonian has the kinetic term with a nontrivial Hermitian metric,

$$
H^{\mathrm{kin}}=\left(e^{\omega^{\dagger}} e^{\omega}\right)_{a b} \bar{\pi}_{a} \pi_{b} \rightarrow\left(e^{\omega^{\dagger}} e^{\omega}\right)^{\bar{k} j} \bar{\pi}_{\bar{k}} \pi_{j} .
$$


The matrices $e^{ \pm \omega}, e^{ \pm \omega^{\dagger}}$ can then be interpreted as the complex vielbeins,

$$
\left(e^{\omega}\right)_{a c} \rightarrow e_{a}^{j}, \quad\left(e^{-\omega}\right)_{c a} \rightarrow e_{j}^{a}, \quad\left(e^{\omega \dagger}\right)_{c a} \rightarrow e_{\bar{a}}^{\bar{j}}, \quad\left(e^{-\omega \dagger}\right)_{a c} \rightarrow e_{\bar{j}}^{\bar{a}}
$$

When $\omega$ is Hermitian, the vielbein matrix $e_{a}^{j}$ is also Hermitian. For generic $\omega$, the vielbein is a generic complex matrix, with the anti-Hermitian part of $\omega$ corresponding to tangent space rotations.

One can note now that the supercharge (2.5) can be rewritten as

$$
Q=\sqrt{2} \psi^{j}\left(\pi_{j}+i \Omega_{j, \bar{b} a} \psi_{a} \bar{\psi}_{b}\right)
$$

with $\Omega_{k, \bar{b} a}$ being the so called Bismut spin connection corresponding to the metric $h=e^{\omega \dagger} e^{\omega}$ and the vielbein $e=e^{\omega}$. $^{2}$

A nontrivial metric introduces a natural covariant measure in the Hilbert space,

$$
\mu=\operatorname{det} h \prod_{j} d z^{j} d \bar{z}^{j}
$$

It is convenient to define $\bar{Q}$ to be Hermitially conjugate to $Q$ with respect to this measure,

$$
\bar{Q}=(\operatorname{det} h)^{-1} Q^{\dagger} \operatorname{det} h,
$$

where $Q^{\dagger}$ is the "naive" Hermitian conjugation.

The supercharges thus obtained exactly coincide with the supercharges (3.26) in ref. [4], if setting there $W=\ln \operatorname{det} h / 4$. These supercharges were obtained from Nöther supercharges of a certain SQM model with a nontrivial superspace Lagrangian $[4,6]$,

$$
L=-\frac{1}{4} h_{j \bar{k}} D Z^{j} \bar{D} \bar{Z}^{\bar{k}}+W(Z, \bar{Z})
$$

where $Z^{j}$ and $\bar{Z}^{j}$ are chiral $d=1$ superfields and $D, \bar{D}$ are supersymmetric covariant derivatives.

As was explained in details in [4], the Hilbert space of the functions $\Psi\left(z^{j}, \bar{z}^{j}, \psi_{a}\right)$ with the measure (2.9) can be mapped onto the space of holomorphic $p$-forms realizing the Dolbeault complex. The supercharges (2.8), (2.10) can then be mapped to the exterior holomorphic derivative operator $\partial$ and its Hermitian conjugate.

As was just mentioned, the supercharges (2.8) and (2.10) correspond to a particular choice of $W$ in the Lagrangian (2.11). One can, however, obtain the model with any $W$ by applying an extra similarity transformation,

$$
Q \rightarrow e^{G} Q e^{-G}
$$

\footnotetext{
${ }^{2}$ The Bismut spin connection is related to the Bismut affine connection $[2,3]$, which is a torsionfull affine connection such that (i) the covariant derivatives of the metric and the complex structure matrix vanish; (ii) the torsions are completely antisymmetric. See $[4,5]$ for all definitions and notations. To avoid a confusion, note also here that the Bismut spin connection does not coincide with the structure $-e_{a}^{l}\left(\partial_{j} e_{l}^{b}\right)$ entering (2.5), but involves extra terms. These terms vanish when multiplying by $\psi^{j} \psi_{a}$. cf. eq. (3.13) of ref. [4].
} 
with $G=W-\frac{1}{4} \ln \operatorname{det} h .^{3}$ This gives what mathematicians call a twisted holomorphic Dolbeault complex.

Another distinguished choice, besides $W=\frac{1}{4} \ln \operatorname{det} h$ corresponding to $G=0$, is $W=-\frac{1}{4} \ln \operatorname{det} h$ corresponding to $G=-\frac{1}{2} \ln \operatorname{det} h$. This amounts to the overall similarity transformation with $\exp \left\{-\omega_{a b} \bar{\psi}_{b} \psi_{a}\right\}$ and gives the antiholomorphic untwisted Dolbeault complex.

In the physical language, this complex describes the dynamics of a Dirac operator in the presence of Abelian gauge field, $A_{M}=\left\{-i \partial_{m} W, i \partial_{\bar{m}} W\right\}$. The models with nonAbelian gauge fields can be obtained from the model (2.11) with $W=0$ by a similarity transformation (2.12) with a matrix-valued $G$.

The supercharge $Q$ can be further rotated with a holomorphic ${ }^{4}$ operator

$$
\exp \left\{\mathcal{B}_{j k} \psi^{j} \psi^{k}+\mathcal{B}_{j k l m} \psi^{j} \psi^{k} \psi^{l} \psi^{m}+\ldots\right\}
$$

One obtains in this way complex sigma models with torsions studied in [8].

\subsection{De Rham complex}

Note first that, representing $\pi_{a}=\left[p_{x}^{(a)}+i p_{y}^{(a)}\right] / \sqrt{2}$ in (1.5) and imposing $d$ constraints $p_{y}^{(a)} \Psi=0$, we obtain the model describing free flat real dynamics. The supercharges are

$$
Q=p_{A} \psi_{A}, \quad \bar{Q}=p_{A} \bar{\psi}_{A} .
$$

(with $\left.p_{A} \equiv p_{x}^{(a)}, A=1, \ldots, d\right)$.

Let us apply now a similarity transformation (2.1) with

$$
R=\omega_{A B} \psi_{A} \bar{\psi}_{B}
$$

When $\omega_{A B}$ is anti-Hermitian, this amounts to a unitary rotation. New nontrivial models are obtained for Hermitian $\omega_{A B}$.

Let first $\omega_{A B}$ be real and symmetric. By the same token as in the complex case, one obtains

$$
Q=\psi_{D}\left(e^{\omega}\right)_{D C}\left[p_{C}-i\left(e^{\omega} \partial_{C} e^{-\omega}\right)_{A B} \psi_{A} \bar{\psi}_{B}\right] \equiv \psi^{M}\left(p_{M}-i \Omega_{M, A B} \psi_{A} \bar{\psi}_{B}\right)
$$

where $\psi^{M}=e_{A}^{M} \psi_{A}$ with the real vielbeins

$$
e_{A}^{M}=\left(e^{\omega}\right)_{A}^{M}, \quad e_{M A}=\left(e^{-\omega}\right)_{M A}
$$

giving the metric

$$
g_{M N}=\left(e^{-2 \omega}\right)_{M N} .
$$

\footnotetext{
${ }^{3}$ For a compact complex manifold, there is no global expression for the metric valid everywhere. One should introduce charts. The expressions like $\ln \operatorname{det} h$ are in fact not nonsingular functions on the manifold, but should be understood as sections of a certain line (Abelian) fiber bundle. Matching the local expressions for these sections in the regions where the charts overlap imposes the restrictions on $W$ associated with the quantization of topological charge. If these restrictions are not fulfilled, supersymmetry is lost [7].

${ }^{4}$ It is holomorphic with respect to fermion variables, but $\mathcal{B}_{j k}$ etc. are arbitrary functions of $z^{j}$ and $\bar{z}^{j}$.
} 
Profiting from anticommutativity of $\psi^{M}$ and $\psi_{A}$, we have expressed the 3-fermion structure in the supercharge via the spin connection

$$
\Omega_{M, A B}=e_{A N}\left(\partial_{M} e_{B}^{N}+\Gamma_{M K}^{N} e_{B}^{K}\right) .
$$

The supercharge (2.16) is well known. It can be mapped to the exterior derivative operator $d$ of the de Rham complex $[9,10]$. The supercharge $\bar{Q}$ is convenient to define as $\bar{Q}=(\operatorname{det} g)^{-1 / 2} Q^{\dagger}(\operatorname{det} g)^{1 / 2}$ such that it is Hermitially conjugate to $Q$ with the Riemann covariant measure $\sqrt{\operatorname{det} g}$. It can be presented as

$$
\bar{Q}=\bar{\psi}^{M}\left(p_{M}-i \Omega_{M, A B} \bar{\psi}_{A} \psi_{B}\right) .
$$

The Lagrangian of this model can be easily written in terms of $d$ real superfields

$$
X^{M}=x^{M}+\theta \psi^{M}+\bar{\psi}^{M} \bar{\theta}+F^{M} \theta \bar{\theta} .
$$

It has the form [11]

$$
L=\frac{1}{2} \int d \theta d \bar{\theta} g_{M N}(X) D X^{M} \bar{D} X^{N} .
$$

The de Rham complex can be deformed by adding the potential, which amounts to a similarity transformation $Q \rightarrow e^{W} Q e^{-W}$ (in contrast to the complex case, here $W$ must be a well-defined scalar function) or adding torsions [8, 12] which amounts to a holomorphic similarity transformation with the operator $\exp \left\{\mathcal{B}_{M N} \psi^{M} \psi^{N}+\cdots\right\}$.

Consider now the case of generic Hermitian $\omega_{A B}$. The supercharges can, again, be represented as in (2.16), but neither the vielbeins (2.17) nor the "metric" (2.18) are real anymore. As is shown in [13], the corresponding Lagrangian has the same form as in (2.22), but with the complex Hermitian $g_{M N}$. Complexity of the metric means that this system cannot thus be interpreted anymore as a de Rham complex. It is something new.

It is further shown in [13] that this new complex can be obtained by a Hamiltonian reduction of the Dolbeault complex for some special complex manifolds whose metric does not depend on imaginary parts of complex coordinates. Indeed, consider a manifold of the complex dimension $d$ with isometries corresponding to the imaginary coordinate shifts. We can then impose $d$ constraints $G_{j} \Psi=\partial \Psi / \partial\left(\operatorname{Im} z^{j}\right)=0$. These constraints commute with the Dolbeault Hamiltonian (allowing for the Hamiltonian reduction) and the supercharges (meaning that the reduced system enjoys the same supersymmetry as the original one). A not so difficult analysis shows that, after such reduction, the supercharge (2.8) (it is convenient to write it in terms of the fermion variables with world indices $\psi^{j}$ ) goes over into (2.16), written in terms of $\psi^{M}, \bar{\psi}^{M}$.

In other words, the model of this type (we called it a quasicomplex sigma model [13]) can be obtained from the basic model (1.5) by a subsequent application of two operations: similarity transformation and Hamiltonian reduction. And, as illustrated in figure 1, the result does not depend on the order in which these operations are performed.

We would also like to note here that the supercharges $Q=\sqrt{2} \psi_{a} \pi_{a}$ and $Q=\psi_{A} p_{A}$ can in principle be rotated by a similarity transformations with antiholomorphic operators like

$$
R=\exp \left\{\mathcal{C}_{A B} \bar{\psi}_{A} \bar{\psi}_{B}\right\} .
$$

Such models were never considered, and it would be interesting to do so. 


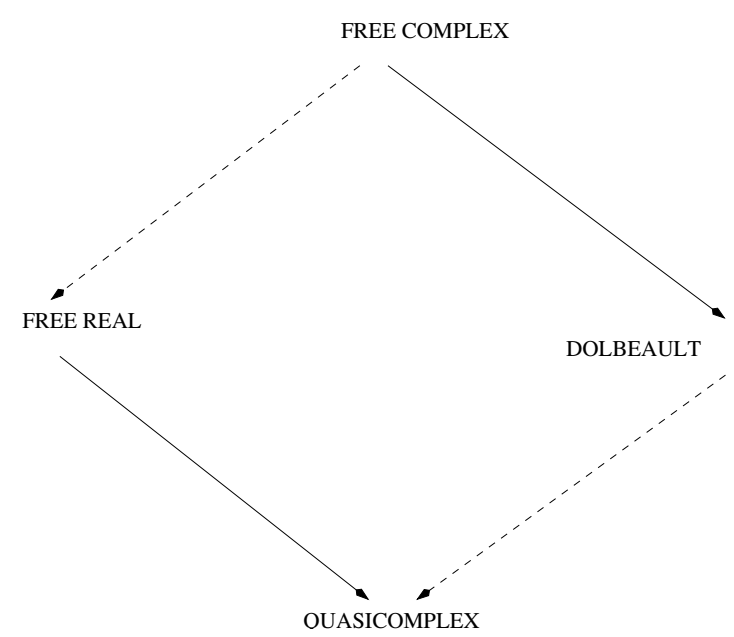

Figure 1. A rhombus of sigma models. The solid arrows stand for a similarity transformation and the dashed arrows - for a Hamiltonian reduction.

\section{$3 \quad$ Extended supersymetries}

An arbitrary similarity transformation (2.1) leaves the supercharge $Q$ nilpotent and hence keeps supersymmetry. For a model with extended supersymmetries, it is not always the case. I.e. the minimal $\mathcal{N}=2$ supersymmetry is always kept, but if we want to preserve extended supersymmetries, the operator of similarity transformation should satisfy certain extra conditions. The same concerns the Hamiltonian reduction procedure. If we want the reduced model to keep all the supersymmetries of the original one, the constraints should commute with all supercharges.

Consider some examples.

\subsection{Kähler sigma models}

Consider the real free dynamics with the supercharges (2.14) and the Hamiltonian $H=$ $p_{A}^{2} / 2$. Assume that the dimension $D$ is even. It is easy to see that one can add extra pairs of nilpotent supercharges whose anticommutator gives the same Hamiltonian and which commute with $Q, \bar{Q}$. They form thereby together with (2.14) an extended supersymmetry algebra. Each such pair of supercharges can be represented as

$$
S=p_{A} I_{A B} \psi_{B}, \quad \bar{S}=p_{A} I_{A B} \bar{\psi}_{B}
$$

where $I_{A B}$ is a real antisymmetric matrix satisfying the condition $I^{2}=-1 .^{5}$

Suppose that there is only one such extra pair. It is convenient to introduce complex coordinates $\left\{z_{a=1, \ldots, D / 2}, \bar{z}_{a=1, \ldots, D / 2}\right\}$ (the eigenvectors of $I$ ), trade $\psi_{A}$ for $\chi_{a \alpha}, \alpha=1,2$ and

\footnotetext{
${ }^{5}$ It is, of course, recognizable as a flat complex structure matrix.
} 
deal with the supercharges ${ }^{6}$

$$
T_{\alpha}=\sqrt{2} \pi_{a} \chi_{a \alpha}, \quad \bar{T}_{\alpha}=\sqrt{2} \bar{\pi}_{a} \bar{\chi}_{a \alpha} .
$$

They represent the following linear combinations of $Q, S, \bar{Q}, \bar{S}$,

$$
T_{1}=\frac{Q+i S}{\sqrt{2}}, \quad T_{2}=\frac{\bar{Q}+i \bar{S}}{\sqrt{2}}, \quad \bar{T}_{1}=\frac{\bar{Q}-i \bar{S}}{\sqrt{2}}, \quad \bar{T}_{2}=\frac{Q-i S}{\sqrt{2}} .
$$

Let us perform now a similarity transformation

$$
T_{\alpha} \rightarrow e^{\omega_{a b} \chi_{a \beta} \bar{\chi}_{b \beta}} T_{\alpha} e^{-\omega_{a b} \chi_{a \beta} \bar{\chi}_{b \beta}}
$$

with a Hermitian $\omega_{a b}$. In the full analogy with (2.5), (2.8), we obtain

$$
T_{\alpha}=\sqrt{2} \chi_{d \alpha}\left(e^{\omega}\right)_{d c}\left[\pi_{c}-i\left(e^{\omega}\right)_{a e}\left(\partial_{c} e^{-\omega}\right)_{e b} \chi_{a \beta} \bar{\chi}_{b \beta}\right]
$$

which can be represented as

$$
T_{\alpha}=\sqrt{2} \chi_{\alpha}^{j}\left(\pi_{j}+i \Omega_{j, \bar{b} a} \chi_{a \beta} \bar{\chi}_{b \beta}\right)
$$

However, in a generic case, the supercharges (3.6) and their conjugates do not form the extended supersymmetry algebra - the anticommutator $\left\{T_{1}, \bar{T}_{2}\right\}$ does not vanish and $\left\{T_{2}, \bar{T}_{2}\right\}$ does not coincide with $\left\{T_{1}, \bar{T}_{1}\right\}$. But, for some special $\omega$ when the metric $e^{\omega^{\dagger}} e^{\omega}$ is Kähler, the $\mathcal{N}=4$ superalgebra holds [14-16]. In this case, $\Omega_{j, \bar{b} a}=e_{\bar{b}}^{\bar{k}} \partial_{j} e_{\bar{k}}^{\bar{a}}$ entering (3.6) are the standard torsionless spin connections.

As is written in (3.3), the supercharge $T_{1}$ is expressed via $Q$ and $S$, while the supercharge $T_{2}$ is expressed via $\bar{Q}$ and $\bar{S}$. This means that the similarity transformation (3.4) of $T_{\alpha}$ corresponds to a rather complicated transformation (not a similarity one) of the "original" supercharges $Q, S$.

Alternatively, one can rotate, as we have seen, the flat supercharge $Q$ with the operator (2.15) to obtain the de Rham supercharge (2.16). If the metric thus obtained is Kähler, the same similarity transformation applied to $S$ gives us the second pair of supercharges, keeping the $\mathcal{N}=4$ supersymmetry. Indeed, the result of such rotation of $S^{\text {flat }}$ is

$$
S^{\text {rotated }}=\psi^{M} I_{M}^{N}\left(p_{N}-i \Omega_{N, A B} \psi_{A} \bar{\psi}_{B}\right) .
$$

An accurate proof of the fact that, for Kähler manifolds, the operators (3.7) and (2.16) together with their conjugates satisfy the same commutation relations of the $\mathcal{N}=4$ superalgebra as the flat supercharges is presented in the appendix.

Note that this similarity transformation of $Q$ and $S$ corresponds to a complicated transformation of $T_{\alpha}$ and $\bar{T}_{\alpha}$.

\footnotetext{
${ }^{6}$ When $D=2$ and $I_{A B}=\epsilon_{A B}$, the explicit conventional form of the combinations entering (3.2) is

$$
\chi_{1}=\frac{1}{\sqrt{2}}\left(\psi_{1}+i \psi_{2}\right), \quad \chi_{2}=\frac{1}{\sqrt{2}}\left(\bar{\psi}_{1}+i \bar{\psi}_{2}\right), \quad \pi=\frac{1}{\sqrt{2}}\left(p_{1}-i p_{2}\right)
$$
}

and complex conjugates. This is trivially generalized to any even $D$, if choosing $I=\operatorname{diag}(\epsilon, \ldots, \epsilon)$. 


\subsection{Hyper-Kähler sigma models}

In flat space of real dimension 4 or multiple integer of $4, D=4 m$, one can write three additional pairs of supercharges,

$$
S^{1,2,3}=p_{A} I_{A B}^{1,2,3} \psi_{B}, \quad \bar{S}^{1,2,3}=p_{A} I_{A B}^{1,2,3} \bar{\psi}_{B} .
$$

associated with three complex structures $I^{1,2,3}$ satisfying the quaternionic algebra.

$$
I^{a} I^{b}=-\delta^{a b}+\epsilon^{a b c} I^{c}
$$

By an orthogonal transformation, one can bring them into a canonical form

$$
\left(I^{a}\right)_{A B}=\operatorname{diag}\left\{-\eta_{\mu \nu}^{a}, \ldots,-\eta_{\mu \nu}^{a}\right\}
$$

where $\eta_{\mu \nu}^{a}$ are 't Hooft symbols.

The supercharges $Q, \bar{Q}, S^{a}, \bar{S}^{a}$ form the $\mathcal{N}=8$ supersymmetry algebra, which the flat Hamiltonian $H=p_{A}^{2} / 2$ thus enjoy. In a special case when the metric of the manifold corresponding to the de Rham supercharges (2.16) obtained after a similarity transformation of the flat supercharge $Q$ is hyper-Kähler, the Hamiltonian thus obtained also admits 3 extra pairs of conserved supercharges [17]. Three of these six extra supercharges are obtained from the flat supercharges $S^{a}$ in (3.8) by the same similarity transformation as the one applied to the supercharge $Q$. Their explicit form is

$$
S_{\text {rotated }}^{a}=\psi^{M} I_{M}^{a}{ }^{N}\left(p_{N}-i \Omega_{N, A B} \psi_{A} \bar{\psi}_{B}\right)
$$

The explicit proof of the fact that, in the hyper-Kähler case, the supercharges (3.11) and their conjugates form together with the supercharge (2.16) and its conjugate the standard $\mathcal{N}=8$ superalgebra is given in the appendix.

Alternatively, in the full analogy with the Kähler case, the supercharges (3.8) together with (2.14) can be rearranged by defining

$$
T_{\alpha}=\left(\gamma_{\mu} p_{\mu}^{k} \chi^{k}\right)_{\alpha}
$$

and their conjugates $\bar{T}_{\alpha}$, In the expression above, $\mu, \alpha=1,2,3,4, k=1, \ldots, m, \gamma_{\mu}$ are Euclidean 4-dimensional $\gamma$-matrices and $\chi_{\alpha}^{k}$ are Dirac 4-component spinors.

One can then rotate $T_{\alpha}$ with a matrix $e^{R}=\exp \left\{\omega_{k q} \chi_{\alpha}^{k} \bar{\chi}_{\alpha}^{q}\right\}$ and, when the metric $e^{\omega^{\dagger}} e^{\omega}$ thus obtained is hyper-Kähler, arrive at the hyper-Kähler supercharges in the form written in [18].

This similarity transformation of $T_{\alpha}$ corresponds to a complicated transformation of $Q, \bar{Q}, S^{a}, \bar{S}^{a}$. On the other hand, the similarity transformation of $Q$ and $S^{a}$ discussed above corresponds to a complicated transformation of $T_{\alpha}, \bar{T}_{\alpha}$.

\subsection{HKT and OKT}

Kähler and hyper-Kähler sigma models represent special cases of the generic de Rham sigma model that admit extra supercharges. There are also special complex Dolbeault 
sigma models admitting extra supersymmetries. In particular, in flat complex space of even complex dimension $d=2 m$, one can add to the supercharges (1.5), the supercharges

$$
S=\sqrt{2} \epsilon_{a b} \psi_{a}^{k} \bar{\pi}_{b}^{k}, \quad \bar{S}=\sqrt{2} \epsilon_{a b} \bar{\psi}_{a}^{k} \pi_{b}^{k}
$$

Performing a similarity transformation (2.1) with a special class of $R$ respecting $\mathcal{N}=4$ supersymmetry, one obtains the so called HKT sigma models [19, 20]. To see that, consider the simplest $d=2$ case. By introducing real and imaginary parts $\psi_{A=1,2,3,4}$ of $\psi_{a=1,2}$, we can bring (1.5), (3.13) to a more familiar form including four pairwise anticommuting Hermitian supercharges [21, 22],

$$
\mathcal{Q}=\psi_{A} p_{A}, \quad \mathcal{S}^{a}=-\eta_{A B}^{a} \psi_{A} p_{B}
$$

where $a=1,2,3$ and $\eta_{A B}^{a}$ are 't Hooft symbols [23].

This looks similar to (3.8), but the variables $\psi_{A}$ are now Hermitian. They satisfy the Clifford algebra, $\left\{\psi_{A}, \psi_{B}\right\}=\delta_{A B}$ and can be mapped to gamma matrices. For our approach, we need, however the holomorphic supercharges $Q, S$ in (1.5), (3.13). It is they who are going to be rotated with a similarity transformation (2.1), the supercharges $\bar{Q}, \bar{S}$ being transformed with $e^{-R^{\dagger}}$. Let us choose $R=g(x) \psi_{a} \bar{\psi}_{a}$. We derive

$$
\begin{aligned}
Q & \rightarrow \sqrt{2} f \psi_{a}\left(\pi_{a}+\frac{i \partial_{a} f}{f} \psi_{c} \bar{\psi}_{c}\right), \\
S & \rightarrow \sqrt{2} f \epsilon_{a b} \psi_{a}\left(\bar{\pi}_{b}+\frac{i \bar{\partial}_{b} f}{f} \psi_{c} \bar{\psi}_{c}\right) .
\end{aligned}
$$

with $f=e^{g}$. The corresponding metric is conformally flat, $d s^{2}=e^{-2 g} d x_{\mu}^{2}$, the simplest HKT metric. ${ }^{7}$ The supercharges (3.15) (derived first in [25]) together with their conjugates satisfy the $\mathcal{N}=4$ superalgebra.

Dolbeault models enjoying $\mathcal{N}=8$ supersymmetry are known as OKT manifolds. Their real dimension is a multiple integer of 8 . Indeed, flat 8 -dimensional space admits 8 anticommuting Hermitian supercharges: the supercharge $\mathcal{Q}=p_{A} \psi_{A}$, where $A=1, \ldots, 8$ and $\psi_{a}$ are now real, and the supercharges $\mathcal{S}^{a=1, \ldots, 7}=\left(\Gamma^{a}\right)_{A B} p_{A} \psi_{B}$, where $\Gamma^{a}$ are 7-dimensional real antisymmetric gamma matrices. One of the convenient representations for the latter is

$$
\Gamma^{1,2,3}=\left(\begin{array}{cc}
-\bar{\eta}^{a} & 0 \\
0 & \bar{\eta}^{a}
\end{array}\right), \quad \Gamma^{4,5,6}=\left(\begin{array}{cc}
0 & \eta^{a} \\
\eta^{a} & 0
\end{array}\right), \quad \Gamma^{7}=\left(\begin{array}{cc}
0 & 1 \\
-1 & 0
\end{array}\right),
$$

\footnotetext{
${ }^{7}$ The formal definition is the following [24]. The manifold is called HKT if it admits three complex structures satisfying (3.9) which are covariantly constant with respect to one and the same affine connection. Generically (and, in particular, for conformally flat 4-dimensional manifolds), this connection (the Bismut connection) involves torsions. In some special cases, the torsions vanish, the Bismut connection boils down to the usual Levy-Civita connection and the HKT (i.e. hyper-Kähler with torsion ) manifolds boil down hyper-Kähler manifolds.
} 
One can also relate the matrix elements in (3.16) to the structure constants of octonion algebra [20] - that is why the name OKT (octonionic Kähler with torsion) was choosen. ${ }^{8}$ The simplest example of a nontrivial OKT manifold is a conformally flat 8-dimensional manifold where the conformal factor $f\left(x^{M}\right)$ represents a harmonic function, i.e. $f\left(x^{M}\right)=$ $1+C\left|x^{M}\right|^{-7}$.

The OKT models represent a particular case of Dolbeault models with extra holomorphic torsions (see [27] for a detailed discussion). Thus, they can be obtained, as discussed above, from the flat models of the corresponding dimension by a similarity transformation of the supercharge $Q$ in (1.5). It remains to be seen whether one can conveniently define in this case three other complex supercharges obtained from the flat ones by the same similarity transformation.

\subsection{Reduced models}

Consider an HKT model on a 4-dimensional conformally flat manifold with the supercharges (3.15). Suppose that the conformal factor $f\left(x^{M}\right)$ does not depend on one of the variables, say, $x^{4}$. It is then straightforward to observe that the operator $\hat{p}_{4}$ commutes with the supercharges and the Hamiltonian and can thus be used to perform Hamiltonian reduction. As a result, we obtain a $\mathcal{N}=4$ supersymmetric QM model describing dynamics on a conformally flat 3 -dimensional manifold. This model $^{9}$ was first constructed in [29] and described in superfield language in [30,31]. Taking a $4 m$ dimensional HKT with the metric not depending on $m$ variables, one obtains a generalised $3 m$-dimensional model considered in [30].

One can, of course, consider many other HKT models (or, in the language of [28], the models with several root $[32](\mathbf{4}, \mathbf{4}, \mathbf{0})$ multiplets) living on manifolds with various isometries. Factorizing over these isometries gives a multitude of models. Hamiltonian reduction of the model in a flat or conformally flat 4-dimensional space with respect to its $\mathrm{U}(1)$ isometry was considered in [33]. One obtains in such a way the $\mathcal{N}=4$ models with an extra magnetic monopole $[29,34]$. For another example, one can take a flat $\mathcal{N}=4$ model endowed with a self-dual instanton field, ${ }^{10}$

$$
\mathcal{A}_{\mu}=\frac{2 \eta_{\mu \nu}^{a} x_{\nu} t^{a}}{x^{2}+\rho^{2}}
$$

\footnotetext{
${ }^{8}$ In contrast to HKT, the matrices (3.16) cannot, of course, satisfy non-associative octonion algebra. Moreover, one cannot choose among the matrices (3.16) three matrices satisfying the quaternionic algebra (3.9). This means that an OKT manifold need not to be an HKT manifold. Note also that one can deform the flat model with breaking $\mathcal{N}=8$ supersymmetry but keeping the $\mathcal{N}=4$ supersymmetry associated with the unity matrix and, say, the matrices $\Gamma^{1,2,3}$. One obtains in this way a class of $\mathcal{N}=4$ models, so called Clifford models that are not $\operatorname{HKT}[6,20,26,27]$.

${ }^{9} \mathrm{~A}(\mathbf{3}, \mathbf{4}, \mathbf{1})$-model in the notation of [28], where the first numeral stands for the number of bosonic dynamic degrees of freedom, the second - the number of fermionic d.o.f. and the third — the number of auxiliary fields in superfield description.

${ }^{10}$ It was mentioned above that a non-Abelian gauge field can be brought about by a matrix-valued similarity transformation. The transformations of this kind that give a self-dual gauge field respect the $\mathcal{N}=4$ supersymmetry of the flat model [25].
} 
The supercharges of this model were presented in ref. [25] in the form

$$
Q_{\alpha}=\left(\sigma_{\mu} \bar{\psi}\right)_{\alpha}\left(p_{\mu}-\mathcal{A}_{\mu}\right), \quad \bar{Q}^{\alpha}=\left(\psi \sigma_{\mu}^{\dagger}\right)^{\alpha}\left(p_{\mu}-\mathcal{A}_{\mu}\right)
$$

with the following conventions (an Euclidean counterpart of the Wess and Bagger notations [35]):

(i) $\left(\sigma_{\mu}\right)_{\alpha \dot{\beta}}=\{i, \boldsymbol{\sigma}\}_{\alpha \dot{\beta}},\left(\sigma_{\mu}^{\dagger}\right)^{\dot{\beta} \alpha}=\{-i, \boldsymbol{\sigma}\}^{\dot{\beta} \alpha}$.

(ii) The fermion variables $\psi_{\dot{\alpha}}$ and $\bar{\psi}^{\dot{\alpha}}=\left(\psi_{\dot{\alpha}}\right)^{\dagger} \quad\left(\left\{\bar{\psi}^{\dot{\alpha}}, \psi_{\dot{\beta}}\right\}=\delta_{\dot{\beta}}^{\dot{\alpha}}\right)$ carry only the dotted indices (in constrast to the supercharges (3.18) having undotted indices; in Euclidean space, the SU(2) groups acting on the dotted and undotted spinors are completely unrelated).

(iii) The indices are raised and lowered with $\epsilon_{\dot{\alpha} \dot{\beta}}=-\epsilon^{\dot{\alpha} \dot{\beta}}$.

One can observe now that the supercharges (3.18) and hence the Hamiltonian commute with the $\mathrm{SU}(2)$ generators,

$$
\hat{L}^{a}=2 t^{a}-i \eta_{\mu \nu}^{a}\left(x_{\mu} \partial_{\nu}+\frac{1}{4} \psi \sigma_{\mu}^{\dagger} \sigma_{\nu} \bar{\psi}\right)
$$

Performing the Hamiltonian reduction with respect to $\hat{L}^{a}$ gives us a $(\mathbf{1}, \mathbf{4}, \mathbf{3})$ model with only one dynamic bosonic degree of freedom. At the distances much larger than the instanton size $\rho$, the Hamiltonian thus obtained should go over to the conformal matrix Hamiltonian derived in [36],

$$
H=\frac{1}{2}\left(p^{2}+\frac{3}{4 x^{2}}\right)+\frac{2 i t^{a}\left(\psi \sigma^{a} \bar{\psi}\right)}{x^{2}} .
$$

In that paper, also a 2-center model not enjoying the rotational symmetry was worked out. Probably, it can also be obtained from a certain known model by applying two operations (similarity transformation and Hamiltonian reduction). It would be interesting to see whether it is the case and, if yes, in a what particular way. The same concerns many particular SQM models with $\mathcal{N}=4$ and $\mathcal{N}=2$ supersymmetries constructed in recent [37,38]. They were constructed using "semi-dynamic" spin variables technique [39-45]. The mathematical structure of these models, their raison d'être is, however, not clear by now. It would be interesting to find out by what particular operations from what particular known models are they obtained.

The OKT models with one or several root $(\mathbf{8}, \mathbf{8}, \mathbf{0})$-multiplets also generate many different models after Hamiltonian reduction. One of them is the beautiful $\mathcal{N}=8(\mathbf{5}, \mathbf{8}$, 3)-model with the metric $d s^{2}=\left(1+C / r^{3}\right)\left(d x^{M}\right)^{2}[46-48] .{ }^{11}$

There are many others.

\footnotetext{
${ }^{11}$ It enjoys $\mathrm{O}(5)=\mathrm{Sp}(4)$ symmetry and has many common features with the $(\mathbf{3}, \mathbf{4}, \mathbf{1})$ model of ref. [29] which is 3 -dimensional and knows about $\mathrm{O}(3)=\mathrm{Sp}(2)$. That is why we called this type of sigma models symplectic.
} 


\section{Gauge models}

It is known since Dirac that gauge theories can be interpreted as Hamiltonian systems involving first class constraints, the operators $\hat{G}^{a}$ commuting with the Hamiltonian. One then performs a Hamiltonian reduction with respect to $\hat{G}^{a}$ such that the Hilbert space of the large system is reduced to the small physical Hilbert space including only the wave functions annihilated under the action of $G^{a}$. This is rather similar to the ideology of the present paper, but there is also an important dictinction.

Consider a supersymmetric field theory and assume that nothing depends on spatial coordinates (such dimensional reduction is, of course, a variety of Hamiltonian reduction). We obtain a certain quantum mechanical system. To give a nontrivial enough but not too complicated example, consider the dimensionally reduced $(2+1)$-dimensional supersymmetric Yang-Mills model with SU(2) gauge group. The supercharges of the model are

$$
Q=\Pi_{-}^{a} \psi^{a}+i B^{a} \bar{\psi}^{a}, \quad \bar{Q}=\Pi_{+}^{a} \bar{\psi}^{a}-i B^{a} \psi^{a},
$$

where $\Pi_{ \pm}^{a}=\Pi_{1}^{a} \pm i \Pi_{2}^{a}$ are holomorphic combinations of canonical momenta, $\psi^{a}$ and $\bar{\psi}^{a}$ are canonically conjugated fermion variables, and

$$
B^{a}=\epsilon^{a b c} \epsilon_{j k} A_{j}^{b} A_{k}^{c}=-\frac{i}{2} \epsilon^{a b c} A_{-}^{b} A_{+}^{c}
$$

is the non-Abelian magnetic field strength. The coupling constant dependence is suppressed by choosing proper units.

The Hamiltonian $H=\{\bar{Q}, Q\}$ has the form

$$
H=\frac{1}{2}\left(\Pi_{j}^{a}\right)^{2}+\frac{1}{4}\left[\left(A_{j}^{a} A_{j}^{a}\right)^{2}-A_{j}^{a} A_{k}^{a} A_{j}^{b} A_{k}^{b}\right]+\frac{i \epsilon^{a b c}}{2}\left[\bar{\psi}^{a} \bar{\psi}^{b} A_{+}^{c}+\psi^{a} \psi^{b} A_{-}^{c}\right]
$$

Note, however, that the supercharges written in (4.1) are not nilpotent. One easily derives $Q^{2}=A_{-}^{a} \hat{G}^{a}$, where

$$
\hat{G}^{a}=\epsilon^{a b c}\left(A_{j}^{b} \Pi_{j}^{c}-i \psi^{b} \bar{\psi}^{c}\right)
$$

are Gauss law constraints - generators of gauge transformations. If we want to keep supersymmetry, one should perform the Hamiltonian reduction and impose the constraint $\hat{G}^{a} \Psi=0$.

One can now resolve the constraints, i.e. get rid of three variables on which nothing depends (gauge degrees of freedom) and to write the Hamiltonian in reduced phase space. For field theories, this is practically impossible, but, for quantum mechanical sys-

tems, it is quite feasible. It is convenient to use the polar representation for the vector potential $[49,50]$. In the $(2+1)$-dimensional case, it boils down to

$$
A_{j}^{a}=U_{j k} \Lambda_{k}^{b} V_{b a}
$$

where $U_{j k}(\alpha)$ is an $\mathrm{O}(2)$ matrix describing spatial rotations, $V_{b a}\left(\phi^{a}\right)$ is an $\mathrm{O}(3)$ gauge rotation matrix and $\Lambda_{k}^{b}$ is a quasidiagonal matrix,

$$
\Lambda_{k}^{b}=\left(\begin{array}{lll}
a & 0 & 0 \\
0 & b & 0
\end{array}\right) \text {. }
$$


Choosing the gauge $V_{b a}=\delta_{b a}$, we are left with just three gauge invariant bosonic variables: $a, b, \alpha$. In addition, reduced phase space inherits all three complex fermion variables $\psi^{a}, \bar{\psi}^{a}$. The explicit expressions for the reduced supercharges and Hamiltonian are rather complicated [51]. We will present here only the supercharges.

$$
\begin{aligned}
Q^{\mathrm{cov}}=e^{-i \alpha} g_{0} & {\left[\psi^{1}\left(p_{a}-i \frac{a p_{\alpha}+b J^{3}}{a^{2}-b^{2}}\right)+\psi^{2}\left(-i p_{b}+\frac{b p_{\alpha}+a J^{3}}{a^{2}-b^{2}}\right)\right.} \\
& \left.-\psi^{3}\left(\frac{J^{2}}{a}+\frac{i J^{1}}{b}\right)\right]+\frac{i a b}{g_{0}} \bar{\psi}^{3} \\
\bar{Q}^{\mathrm{cov}}=g_{0} e^{i \alpha} & {\left[\bar{\psi}^{1}\left(p_{a}+i \frac{a p_{\alpha}+b J^{3}}{a^{2}-b^{2}}\right)+\bar{\psi}^{2}\left(i p_{b}+\frac{b p_{\alpha}+a J^{3}}{a^{2}-b^{2}}\right)\right.} \\
& \left.-\bar{\psi}^{3}\left(\frac{J^{2}}{a}-\frac{i J^{1}}{b}\right)\right]-\frac{i a b}{g_{0}} \psi^{3},
\end{aligned}
$$

where $J^{a}=i \epsilon^{a b c} \psi^{b} \bar{\psi}^{c}$.

This is a kind of sigma model, the metric in the space $\{a, b, \alpha\}$ induced by the flat metric in the space $\left\{A_{j}^{a}\right\}$ being nontrivial. The configuration space involves a complex fermion variable for each bosonic variable, i.e. the number of degrees of freedom is the same as for the de Rham complex. But it is not a de Rham system: for the latter the fermion charge is conserved, while the supercharge $Q$ in (4.7) involves the terms $\propto \bar{\psi}$ on top of the terms $\propto \psi$.

If our conjecture is true, the supercharges (4.7) can be obtained by a similarity transformation and Hamiltonian reduction from a free system. However, in this case, a pure similarity transformation of the system (2.14) of real dimension 3 would probably be not sufficient. Indeed, the only imaginable to us way to obtain the term $\propto \bar{\psi}^{3}$ out of a "flat" supercharge like $p_{a} \psi^{(a)}+p_{b} \psi^{(b)}+p_{\alpha} \psi^{(\alpha)}$ is applying an antiholomorphic transformation (2.23). But such a transformation can generate only the terms $\sim \bar{\psi}$ that multiply canonical momenta and in addition the unwanted terms $\propto \psi \bar{\psi} \bar{\psi}$.

Thus, to derive (4.7), one should start from a free system of larger dimension. Indeed, as was discussed above, the system (4.7) can be obtained by a Hamiltonian reduction of a more simply looking system (4.1), (4.3) with extended phase space. But, in constrast to the examples with Hamiltonian reduction discussed in the previous section, such extended system is not supersymmetric - this is the distinction that we were talking about. The absence of supersymmetry in the large system can be traced back to the fact that, when writing (4.1), (4.3), we have already partially fixed the gauge (Wess-Zumino gauge) and got rid of some number of components in the spinor superfield $\Gamma_{\alpha}$ describing $3 D$ SYM theory. Supersymmetry is broken by such partial gauge fixing and is restored when the gauge is fixed completely. The question is thus reduced to the question whether this large SQM system involving all components of $\Gamma_{\alpha}$ can be obtained by our recipe. We hope to address it in later studies.

The same set of question can be asked to a system obtained by the dimensional reduction of (3+1)-dimensional, (5+1)-dimensional, or (9+1)-dimensional SYM theory with or without extra matter multiplets. For example, pure $(3+1)$ SYM theory involves $3 \cdot 3-3=6$ 
gauge invariant variables. The explicit expressions for its reduced supercharges and Hamiltonian are given in ref. [16].

\section{$5 \quad$ Field theories}

A natural question to ask is whether our conjecture formulated for SQM systems works also for field theories.

The fast answer that it should, because quantum field theory is nothing but a SQM system with an infinite number of degrees of freedom. The devil is, as usual, in the details.

Consider the simplest example - the $4 \mathrm{D}$ Wess-Zumino model. To make the things still simpler, let it be free massless WZ model with the Lagrangian

$$
L=\int d \boldsymbol{x}\left[\partial_{\mu} \bar{\phi} \partial_{\mu} \phi+i \psi \sigma_{\mu} \partial_{\mu} \bar{\psi}\right]
$$

where $\psi^{\alpha}, \bar{\psi}^{\dot{\alpha}}$ are complex conjugate Minkowskian Weyl spinors and $\left(\sigma_{\mu}\right)_{\alpha \dot{\beta}}=\left(1, \sigma_{j}\right)_{\alpha \dot{\beta}}{ }^{12}$ The corresponding Hamiltonian

$$
H=\int d \mathbf{x}\left[\bar{\Pi} \Pi+\partial_{j} \bar{\phi} \partial_{j} \phi-i \psi \sigma_{j} \partial_{j} \bar{\psi}\right]
$$

is supersymmetric. There is the Weyl doublet of supercharges,

$$
\begin{aligned}
Q_{\alpha} & =\sqrt{2} \int d \mathbf{x}\left[\Pi \psi_{\alpha}+\partial_{j} \bar{\phi}\left(\sigma_{j}\right)_{\alpha \dot{\gamma}} \delta^{\dot{\gamma} \gamma} \psi_{\gamma}\right] \\
\bar{Q}_{\dot{\alpha}} & =\sqrt{2} \int d \mathbf{x}\left[\bar{\Pi} \bar{\psi}_{\dot{\alpha}}+\left(\partial_{j} \phi\right) \bar{\psi}_{\dot{\gamma}} \delta^{\dot{\gamma} \gamma}\left(\sigma_{j}\right)_{\gamma \dot{\alpha}}\right]
\end{aligned}
$$

They satisfy the algebra

$$
\left\{Q_{\alpha}, \bar{Q}_{\dot{\alpha}}\right\}=2\left(\sigma_{\mu}\right)_{\alpha \dot{\alpha}} P_{\mu}=2\left[\delta_{\alpha \dot{\alpha}} H+\left(\sigma_{j}\right)_{\alpha \dot{\alpha}} P_{j}\right],
$$

where $\boldsymbol{P}$ is the 3 -momentum operator.

Put the system in a finite box of size $L$, which we set to 1 , and expand $\phi(\mathbf{x}), \psi(\mathbf{x})$ in the Fourier series,

$$
\begin{array}{rlrl}
\phi(\mathbf{x}) & =\sum_{\mathbf{n}} \phi_{\mathbf{n}} e^{2 \pi i \mathbf{n} \mathbf{x}}, & \psi(\mathbf{x}) & =\sum_{\mathbf{n}} \psi_{\mathbf{n}} e^{2 \pi i \mathbf{n} \mathbf{x}} \\
\bar{\phi}(\mathbf{x}) & =\sum_{\mathbf{n}} \bar{\phi}_{\mathbf{n}} e^{-2 \pi i \mathbf{n} \mathbf{x}}, & \bar{\psi}(\mathbf{x})=\sum_{\mathbf{n}} \bar{\psi}_{\mathbf{n}} e^{-2 \pi i \mathbf{n} \mathbf{x}}
\end{array}
$$

The Hamiltonian (5.2) is expressed via the modes as follows,

$$
H=\sum_{\mathbf{n}}\left[\bar{\Pi}_{\mathbf{n}} \Pi_{\mathbf{n}}+(2 \pi \mathbf{n})^{2} \bar{\phi}_{\mathbf{n}} \phi_{\mathbf{n}}-2 \pi n_{j} \psi_{\mathbf{n}} \sigma_{j} \bar{\psi}_{\mathbf{n}}\right]
$$

\footnotetext{
${ }^{12}$ Our conventions are almost the same as in ref. [35], but the metric is chosen with the opposite sign, $\eta_{\mu \nu}=\operatorname{diag}(1,-1,-1,-1)$.
} 
$\left(\Pi_{\mathbf{n}}=\dot{\bar{\phi}}_{\mathbf{n}}\right)$ and the supercharges are

$$
\begin{aligned}
Q_{\alpha} & =\sqrt{2} \sum_{\mathbf{n}}\left[\Pi_{\mathbf{n}} \psi_{\alpha \mathbf{n}}-2 \pi i n_{j}\left(\sigma_{j}\right)_{\alpha \beta} \psi_{\beta \mathbf{n}} \bar{\phi}_{\mathbf{n}}\right], \\
\bar{Q}_{\alpha} & =\sqrt{2} \sum_{\mathbf{n}}\left[\bar{\Pi}_{\mathbf{n}} \bar{\psi}_{\alpha \mathbf{n}}+2 \pi i n_{j} \bar{\psi}_{\beta \mathbf{n}}\left(\sigma_{j}\right)_{\beta \alpha} \phi_{\mathbf{n}}\right]
\end{aligned}
$$

(The finite box breaks Lorentz invariance and there is no point to distinguish the usual and dotted indices anymore).

This is an SQM model with an infinite number of degrees of freedom, indeed. One can observe, however, that from the SQM viewpoint,

- it is not a basic system (3.2) as, besides the terms $\propto \Pi \psi$, the supercharges involve extra terms.

- the supercharges (5.7) satisfy not the standard $\mathcal{N}=4$ superalgebra, but the algebra (5.4) involving the 3 -momentum playing the role of a central charge.

Furthermore, the supercharges (5.7) do not seem to be related to the basic supercharges $\sum_{\mathbf{n}} \Pi_{\mathbf{n}} \psi_{\alpha \mathbf{n}}, \sum_{\mathbf{n}} \bar{\Pi}_{\mathbf{n}} \bar{\psi}_{\alpha \mathbf{n}}$ by a similarity transformation.

It is still possible to write down a similarity transformation of the de Rham free (in the SQM sense) supercharge $\mathcal{Q}=p_{A} \psi_{A}$ such that the anticommutator of the transformed supercharge $\mathcal{Q}$ and its conjugate would give (5.6).

Consider one particular term $H_{\mathbf{n}}$ in the sum (5.6). Let first $\mathbf{n} \neq 0$. One can observe that the matrix $n_{j} \sigma_{j}$ has two eigenvalues $\lambda_{1,2}= \pm \sqrt{\mathbf{n}^{2}}$. If denoting by $\chi_{\mathbf{n}}^{1,2}$ the corresponding normalized eigenvectors, one can represent

$$
n_{j} \psi_{\mathbf{n}} \sigma_{j} \bar{\psi}_{\mathbf{n}}=\sqrt{\mathbf{n}^{2}}\left(\chi_{\mathbf{n}}^{1} \bar{\chi}_{\mathbf{n}}^{1}-\chi_{\mathbf{n}}^{2} \bar{\chi}_{\mathbf{n}}^{2}\right)
$$

Then one can define

$$
\mathcal{Q}_{\mathbf{n}}=\chi_{\mathbf{n}}^{1}\left(P_{\mathbf{n}}^{1}+2 i \pi f_{\mathbf{n}}^{1} \sqrt{\mathbf{n}^{2}}\right)+\chi_{\mathbf{n}}^{2}\left(P_{\mathbf{n}}^{2}-2 i \pi f_{\mathbf{n}}^{2} \sqrt{\mathbf{n}^{2}}\right)
$$

$\left(P_{\mathbf{n}}^{1,2} / \sqrt{2}\right.$ and $f_{\mathbf{n}}^{1,2} / \sqrt{2}$ being the real and imaginary parts of $\Pi_{\mathbf{n}}$ and $\left.\phi_{\mathbf{n}}\right)$. The operator $(5.9)$ is nilpotent and

$$
\left\{\mathcal{Q}_{\mathbf{n}}, \overline{\mathcal{Q}}_{\mathbf{n}}\right\}=2 H_{\mathbf{n}} .
$$

This holds also in the case of degenerate eigenvalues, $\mathbf{n}=0$, if choosing for $\chi_{\mathbf{n}}^{1,2}$ arbitrary orthonormal vectors.

Obviously, the full supercharge

$$
\mathcal{Q}=\sum_{\mathbf{n}} \mathcal{Q}_{\mathbf{n}}
$$

is also nilpotent, and $\{\mathcal{Q}, \overline{\mathcal{Q}}\} / 2$ gives the Hamiltonian (5.6). In fact, this model represents a multidimensional (with an infinity of degrees of freedom) generalization of the model (1.1), (1.2) with the superpotential

$$
W=\sum_{\mathbf{n}} \pi \sqrt{\mathbf{n}^{2}}\left[\left(f_{\mathbf{n}}^{1}\right)^{2}-\left(f_{\mathbf{n}}^{2}\right)^{2}\right]
$$


Hence, the supercharge (5.11) can be related to the "free" supercharge

$$
\mathcal{Q}^{(0)}=\sum_{\mathbf{n}}\left(P_{\mathbf{n}}^{1} \chi_{\mathbf{n}}^{1}+P_{\mathbf{n}}^{2} \chi_{\mathbf{n}}^{2}\right)
$$

by the similarity transformation (1.6).

Thus, we showed that, when expanded over the modes, the field theory (5.1) can be obtained by a similarity transformation from the "free" supercharge (5.13), as it should according to our conjecture.

The problem, however, is that this transformation and both the supercharges (5.13) and (5.9) are highly nonlocal. None of them does have therefore a lot of physical sense.

It might be more reasonable to treat the Lorentz-invariant model (5.1) as the free one and ask whether the supercharges of the interacting WZ model could be obtained from the free supercharges (5.3) by a similarity transformation. Unfortunately, the answer to this question seems to be negative.

Indeed, the interacting WZ supercharges are obtained from the free supercharges (5.3) by adding the following extra terms,

$$
\begin{aligned}
& Q_{\alpha}^{\text {int }}=Q_{\alpha}^{\text {free }}+i \sqrt{2} \int d \mathbf{x} \mathcal{W}^{\prime}(\bar{\phi}) \delta_{\alpha \dot{\alpha}} \bar{\psi}^{\dot{\alpha}}, \\
& \bar{Q}_{\dot{\alpha}}^{\text {int }}=\bar{Q}_{\dot{\alpha}}^{\text {free }}-i \sqrt{2} \int d \mathbf{x} \mathcal{W}^{\prime}(\phi) \delta_{\dot{\alpha} \alpha} \psi^{\alpha},
\end{aligned}
$$

where $\mathcal{W}(\phi)$ is the WZ superpotential (having nothing to do with (5.12)). And we do not see how to obtain $Q_{\alpha}^{\text {int }}$ out of $Q_{\alpha}^{\text {free }}$ by a similarity transformation. The problem is the same as with the supercharge $Q^{\text {cov }}$ in eq. (4.7). The only known to us way to generate the term $\propto \bar{\psi}$ in the supercharge $Q$ is to apply an antiholomorphic transformation, like in (2.23). But such a transformation would produce the terms where $\bar{\psi}$ is multiplied by $\Pi$ or else the terms $\propto \bar{\psi} \bar{\psi} \psi \ldots$

\section{Discussion and outlook}

Our main point is the

Conjecture. Any SQM model can be related to a free complex model (1.5) by a combination of two operations: (i) similarity transformation of properly chosen complex supercharges and (ii) Hamiltonian reduction.

We have not proven it, but checked in many nontrivial examples. In particular, we discussed nontrivial sigma models with extended supersymmetries and showed that, for the Kähler de Rham sigma models, hyper-Kähler de Rham sigma models, and HKT models, all complex supercharges are derived from the free supercharges by the same similarity transformation.

On the other hand, we have not seen yet that this conjecture also works for gauge SQM models. We noted that this question can be clarified if analyzing the supercharges and the Hamiltonian of gauge models before gauge is fixed such that supersymmetry is realized linearly. 
In section 5, we discussed field theories and found out that, though our recipe seems to work (it works in the simplest case that we analyzed), the similarity transformation turns out to be highly nonlocal and therefore useless.

The last remark is the following. Philosophically, similarity transformations considered in this paper remind the Nicolai map [52]. In both cases, an interacting model is related to a free one. However, the ways they are related are rather different. The Nicolai map is a nonlocal transformation of bosonic variables that renders the functional integral for the index Gaussian allowing one to do it. For the simplest nontrivial SQM model (1.2), it amounts to the change

$$
\dot{x} \pm W^{\prime}(x) \rightarrow \dot{y}
$$

It is not similar to the local transformation of the supercharges studied in this paper, though more meditations in this direction are definitely welcome.

I am indebted to S. Fedoruk and E. Ivanov for many illuminating discussions.

\section{A Kähler and hyper-Kähler superalgebras}

We will prove here some well-known to mathematicians facts [24] in the SQM language understandable to physicists.

We will be interested in extra supersymmetries of the de Rham $(\{\mathbf{1}, \mathbf{2}, \mathbf{1}\})$ sigma models that come into existence when the manifold is Kähler. We are using the method that was used earlier to study extra supersymmetries for the Dolbeault $(\{\mathbf{2}, \mathbf{2}, \mathbf{0}\}))$ models for hyperKähler [21] and HKT [22] manifolds.

We start with reminding

One of the possible definitions. The complex manifold is called Kähler if its complex structure tensor, ${ }^{13}$

$$
I_{M N}=-I_{N M}, \quad I_{M}^{P} I_{P}^{N}=-\delta_{M}^{N}
$$

is covariantly constant,

$$
\mathcal{D}_{P} I_{M N}=\partial_{P} I_{M N}-\Gamma_{P M}^{S} I_{S N}-\Gamma_{P N}^{S} I_{M S}=0 .
$$

Similarly: the manifold is called hyper-Kähler if it admits three different covariantly constant complex structures $I^{a}$ satisfying the quaternionic algebra (3.9).

We will prove now two theorems.

Theorem 1. If the manifold is Kähler, the supercharges (2.16), (3.7) and their conjugates satisfy the $\mathcal{N}=4$ superalgebra with the only nonvanishing anticommutators

$$
\{Q, \bar{Q}\}=\{S, \bar{S}\}
$$

\footnotetext{
${ }^{13}$ For the manifold to be genuinely complex and not just almost complex, the tensor $I_{M N}$ should satisfy besides (A.1) also a certain integrability condition. But if the tensor $I$ is covariantly constant, this condition is satisfied automatically.
} 
Proof. (i) Nilpotency of $Q, S$ and the property $\{Q, S\}=0$ follow from the proven above fact that $Q$ and $S$ are obtained by the same similarity transformation of the correponding flat supercharges and the validity of the $\mathcal{N}=4$ superalgebra for the latter.

(ii) To deal with the commutators like $\{Q, \bar{S}\}$, introduce the operators

$$
F_{+}=\frac{1}{2} I_{M N} \bar{\psi}^{M} \bar{\psi}^{N}, \quad F_{-}=\frac{1}{2} I_{M N} \psi^{M} \psi^{N} .
$$

Their commutator gives the fermion charge operator, $F_{0}=\psi_{M} \bar{\psi}^{M}$. The operators $\left\{F_{-}, F_{0}, F_{+}\right\}$form an $\mathrm{SU}(2)$ triplet.

(iii) Consider now the commutator $\left[Q, F_{+}\right]$. Capitalizing on the scalar nature of $F_{+}$, one can upgrade the ordinary derivatives in the combination $\partial_{M}+\Omega_{M, A B} \psi_{A} \bar{\psi}_{B}$ to the covariant ones, $\partial_{M} \rightarrow \mathcal{D}_{M}$. The supercharge (2.16) acquires then the form $-i \psi^{M} \nabla_{M}$, where $\nabla_{M}$ is the full covariant derivative involving also the fermion (spinor) part.

Then one notes that

$$
\nabla_{M} \bar{\psi}^{N}=\left(\mathcal{D}_{M} e_{A}^{N}\right) \bar{\psi}_{A}-\Omega_{M, B A} e_{B}^{N} \bar{\psi}_{A}=0
$$

and uses the condition (A.2) that the manifold is Kähler to derive

$$
\begin{aligned}
{\left[Q, F_{+}\right] } & =i \bar{\psi}^{Q} I_{Q}^{M} \nabla_{M}=-\bar{S}, & & {\left[\bar{Q}, F_{-}\right]=-S, } \\
{\left[S, F_{+}\right] } & =\bar{Q}, & {\left[\bar{S}, F_{-}\right] } & =Q
\end{aligned}
$$

(the commutators $\left[Q, F_{-}\right]$and $\left[\bar{Q}, F_{+}\right]$vanish). The vanishing of $\{Q, \bar{S}\}=\left\{Q,\left[F_{+}, Q\right]\right\}$ follows from nilpotency of $Q$ and the Jacobi identity.

(iv) The anticommutator $\{S, \bar{S}\}=\left\{S,\left[F_{+}, Q\right]\right\}$ is reduced to the anticommutator $\left\{\left[S, F_{+}\right], Q\right\}=\{\bar{Q}, Q\}$ by the Jacobi identity.

Theorem 2. If the manifold is hyper-Kähler, the supercharges (2.16), (3.11) and their conjugates satisfy the $\mathcal{N}=8$ superalgebra with the only nonvanishing anticommutators

$$
\{Q, \bar{Q}\}=\left\{S^{1}, \bar{S}^{1}\right\}=\left\{S^{2}, \bar{S}^{2}\right\}=\left\{S^{3}, \bar{S}^{3}\right\} .
$$

Proof. (i) Bearing in mind the results of the previous theorem, we have only to prove that $\left\{S^{a}, S^{b}\right\}=0$ and $\left\{S^{a}, \bar{S}^{b}\right\}=0$ when $a \neq b$. The first equality follows from the fact that all $S^{a}$ are obtained from the flat holomorphic supercharges in (3.8) by one and the same similarity transformation.

(ii) To calculate $\left\{S^{a}, \bar{S}^{b}\right\}$, introduce the operators

$$
F_{+}^{a}=\frac{1}{2} I_{M N}^{a} \bar{\psi}^{M} \bar{\psi}^{N}, \quad F_{-}^{a}=\frac{1}{2} I_{M N}^{a} \psi^{M} \psi^{N} .
$$

The same reasoning as above and the quaternionic algebra (3.9) allow one to derive

$$
\left[S^{a}, F_{+}^{b}\right]=\delta^{a b} \bar{Q}-\epsilon^{a b c} \bar{S}^{c}, \quad\left[\bar{S}^{a}, F_{-}^{b}\right]=\delta^{a b} Q-\epsilon^{a b c} S^{c}
$$

and $\left[S^{a}, F_{-}^{b}\right]=\left[\bar{S}^{a}, F_{+}^{b}\right]=0$. Then e.g. $\left\{S^{1}, \bar{S}^{2}\right\}=\left\{S^{1},\left[S^{1}, F_{+}^{3}\right]\right\}$, which vanishes due to nilpotency of $S_{1}$ and the Jacobi identity. 
Open Access. This article is distributed under the terms of the Creative Commons Attribution License which permits any use, distribution and reproduction in any medium, provided the original author(s) and source are credited.

\section{References}

[1] E. Witten, Dynamical breaking of supersymmetry, Nucl. Phys. B 188 (1981) 513 [inSPIRE].

[2] N. Mavromatos, A note on the Atiyah-Singer index theorem for manifolds with totally antisymmetric $h$ torsion, J. Phys. A 21 (1988) 2279 [InSPIRE].

[3] J.-M. Bismut, A local index theorem for non-Kähler manifolds, Math. Ann. 284 (1989) 681.

[4] E. Ivanov and A. Smilga, Dirac operator on complex manifolds and supersymmetric quantum mechanics, Int. J. Mod. Phys. A 27 (2012) 1230024 [arXiv:1012.2069] [InSPIRE].

[5] A.V. Smilga, Supersymmetric proof of the Hirzebruch-Riemann-Roch theorem for non-Kähler manifolds, SIGMA 8 (2012) 003 [arXiv:1109.2867] [INSPIRE].

[6] C. Hull, The geometry of supersymmetric quantum mechanics, hep-th/9910028 [INSPIRE].

[7] A.V. Smilga, Noninteger flux - why it does not work, J. Math. Phys. 53 (2012) 042103 [arXiv: 1104.3986] [INSPIRE].

[8] S. Fedoruk, E. Ivanov and A. Smilga, Real and complex supersymmetric $D=1 \sigma$-models with torsions, Int. J. Mod. Phys. A 27 (2012) 1250146 [arXiv:1204.4105] [INSPIRE].

[9] E.Witten, Constraints on supersymmetry breaking, Nucl. Phys. B 202 (1982) 253.

[10] E. Witten, Supersymmetry and Morse theory, J. Diff. Geom. 17 (1982) 661 [InSPIRE].

[11] D.Z. Freedman and P. Townsend, Antisymmetric tensor gauge theories and nonlinear

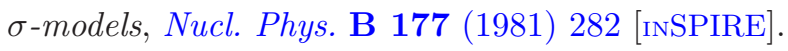

[12] V. Mathai and S. Wu, Analytic torsion for twisted de Rham complexes, J. Diff. Geom. 88 (2011) 297 [arXiv:0810.4204] [INSPIRE].

[13] E. Ivanov and A. Smilga, Quasicomplex $N=2, D=1$ supersymmetric $\sigma$-models, arXiv:1302.2902 [INSPIRE].

[14] A. Davis, A. Macfarlane, P. Popat and J. van Holten, The quantum mechanics of the supersymmetric nonlinear $\sigma$-model, J. Phys. A 17 (1984) 2945 [INSPIRE].

[15] A. Macfarlane and P. Popat, The quantum mechanics of the $N=2$ extended supersymmetric nonlinear $\sigma$-model, J. Phys. A 17 (1984) 2955 [INSPIRE].

[16] A.V. Smilga, How to quantize supersymmetric theories, Nucl. Phys. B 292 (1987) 363 [INSPIRE].

[17] L. Álvarez-Gaumé and D.Z. Freedman, Geometrical Structure and Ultraviolet Finiteness in the Supersymmetric $\sigma$-model, Commun. Math. Phys. 80 (1981) 443 [inSPIRE].

[18] J. Figueroa-O'Farrill, C. Köhl and B.J. Spence, Supersymmetry and the cohomology of (hyper)Kähler manifolds, Nucl. Phys. B 503 (1997) 614 [hep-th/9705161] [INSPIRE].

[19] P.S. Howe and G. Papadopoulos, Twistor spaces for HKT manifolds, Phys. Lett. B 379 (1996) 80 [hep-th/9602108] [INSPIRE].

[20] G. Gibbons, G. Papadopoulos and K. Stelle, HKT and OKT geometries on soliton black hole moduli spaces, Nucl. Phys. B 508 (1997) 623 [hep-th/9706207] [INSPIRE]. 
[21] A. Kirchberg, J. Lange and A. Wipf, Extended supersymmetries and the Dirac operator, Annals Phys. 315 (2005) 467 [hep-th/0401134] [INSPIRE].

[22] A. Smilga, Supercharges in the HKT supersymmetric $\sigma$-models, J. Math. Phys. 53 (2012) 122105 [arXiv:1209.0539] [INSPIRE].

[23] G. 't Hooft, Computation of the quantum effects due to a four-dimensional pseudoparticle, Phys. Rev. D 14 (1976) 3432 [Erratum ibid. D 18 (1978) 2199] [INSPIRE].

[24] M. Verbitsky, HyperKähler manifolds with torsion, supersymmetry and Hodge theory, Asian J. Math. 6 (2002) 679 [math/0112215].

[25] M. Konyushikhin and A.V. Smilga, Self-duality and supersymmetry, Phys. Lett. B 689 (2010) 95 [arXiv: 0910.5162] [INSPIRE].

[26] F. Delduc and E. Ivanov, $N=4$ mechanics of general $(4,4,0)$ multiplets, Nucl. Phys. B 855 (2012) 815 [arXiv:1107.1429] [inSPIRE].

[27] S.A. Fedoruk, E.A. Ivanov and A.V. Smilga, Sigma models with $\mathcal{N}=4$ root multiplets: explicit examples of HKT, CKT, and OKT geometries, in preparation.

[28] A. Pashnev and F. Toppan, On the classification of $N$ extended supersymmetric quantum mechanical systems, J. Math. Phys. 42 (2001) 5257 [hep-th/0010135] [INSPIRE].

[29] A.V. Smilga, Perturbative corrections to effective zero mode hamiltonian in supersymmetric QED, Nucl. Phys. B 291 (1987) 241 [INSPIRE].

[30] E. Ivanov and A.V. Smilga, Supersymmetric gauge quantum mechanics: Superfield description, Phys. Lett. B 257 (1991) 79 [InSPIRE].

[31] V. Berezovoj and A. Pashnev, Three-dimensional $N=4$ extended supersymmetrical quantum mechanics, Class. Quant. Grav. 8 (1991) 2141 [InSPIRE].

[32] M. Faux and S.J. Gates Jr., Adinkras: a graphical technology for supersymmetric representation theory, Phys. Rev. D 71 (2005) 065002 [hep-th/0408004] [INSPIRE].

[33] S. Bellucci, A. Nersessian and A. Yeranyan, Hamiltonian reduction and supersymmetric mechanics with Dirac monopole, Phys. Rev. D 74 (2006) 065022 [hep-th/0606152] [INSPIRE].

[34] M. de Crombrugghe and V. Rittenberg, Supersymmetric quantum mechanics, Annals Phys. 151 (1983) 99 [inSPIRE].

[35] J. Wess and J. Bagger, Supersymmetry and Supergravity, Princeton University Press, Princeton U.S.A. (1983).

[36] S. Fedoruk, E. Ivanov and O. Lechtenfeld, Nahm equations in supersymmetric mechanics, JHEP 06 (2012) 147 [arXiv: 1204.4474] [INSPIRE].

[37] S. Bellucci, N. Kozyrev, S. Krivonos and A. Sutulin, $N=4$ chiral supermultiplet interacting with a magnetic field, Phys. Rev. D 85 (2012) 065024 [arXiv:1112.0763] [INSPIRE].

[38] N. Kozyrev, S. Krivonos and O. Lechtenfeld, $N=2$ supersymmetric $S^{2} \rightarrow C P^{3} \rightarrow S^{4}$ fibration viewed as superparticle mechanics, J. Phys. Conf. Ser. 411 (2013) 012019 [arXiv: 1210.4587] [INSPIRE].

[39] A. Alekseev, L. Faddeev and S.L. Shatashvili, Quantization of symplectic orbits of compact Lie groups by means of the functional integral, J. Geom. Phys. 5 (1988) 391 [INSPIRE]. 
[40] M.R. Douglas and V.A. Kazakov, Large- $N$ phase transition in continuum $Q C D$ in two-dimensions, Phys. Lett. B 319 (1993) 219 [hep-th/9305047] [INSPIRE].

[41] A.P. Polychronakos, Integrable systems from gauged matrix models, Phys. Lett. B 266 (1991) 29 [INSPIRE].

[42] S. Fedoruk, E. Ivanov and O. Lechtenfeld, Supersymmetric Calogero models by gauging, Phys. Rev. D 79 (2009) 105015 [arXiv:0812.4276] [INSPIRE].

[43] S. Fedoruk, E. Ivanov and O. Lechtenfeld, $\operatorname{OSp}(4 \mid 2)$ superconformal mechanics, JHEP 08 (2009) 081 [arXiv:0905.4951] [INSPIRE].

[44] S. Bellucci, S. Krivonos and A. Sutulin, Three dimensional $N=4$ supersymmetric mechanics with Wu-Yang monopole, Phys. Rev. D 81 (2010) 105026 [arXiv:0911.3257] [INSPIRE].

[45] E.A. Ivanov, M.A. Konyushikhin and A.V. Smilga, SQM with non-Abelian self-dual fields: harmonic superspace description, JHEP 05 (2010) 033 [arXiv:0912.3289] [INSPIRE].

[46] D.-E. Diaconescu and R. Entin, A nonrenormalization theorem for the $D=1, N=8$ vector multiplet, Phys. Rev. D 56 (1997) 8045 [hep-th/9706059] [INSPIRE].

[47] A.V. Smilga, Born-Oppenheimer corrections to the effective zero mode Hamiltonian in SYM theory, JHEP 04 (2002) 054 [hep-th/0201048] [INSPIRE].

[48] E. Ivanov and A.V. Smilga, Symplectic $\sigma$-models in superspace, Nucl. Phys. B 694 (2004) 473 [hep-th/0402041] [INSPIRE].

[49] G. Asatrian and G. Savvidy, Configuration manifold of Yang-Mills classical mechanics, Phys. Lett. A 99 (1983) 290 [inSPIRE].

[50] Y. Simonov, QCD hamiltonian in the polar representation, Yad. Fiz. 41 (1985) 1311 [INSPIRE].

[51] A. Smilga, Once more on the Witten index of $3 d$ supersymmetric YM-CS theory, JHEP 05 (2012) 103 [arXiv:1202.6566] [INSPIRE].

[52] H. Nicolai, Supersymmetry and functional integration measures, Nucl. Phys. B 176 (1980) 419 [inSPIRE]. 